\title{
Assessment of inter- and intra-fractional volume of bladder and body contour by mega-voltage computed tomography in helical tomotherapy for pelvic malignancy
}

\author{
Sunghyun Kim, MD, Sei Hwan You, MD, Young Ju Eum, BA, RTT \\ Department of Radiation Oncology, Yonsei University Wonju College of Medicine, Wonju, Korea
}

\begin{abstract}
Purpose: We describe the daily bladder volume change observed by mega-voltage computed tomography (MVCT) during pelvic radiotherapy with potential predictors of increased bladder volume variations.

Materials and Methods: For 41 patients who received pelvic area irradiation, the volumes of bladder and pelvic body contour were measured twice a day with pre- and post-irradiation MVCT from the 1st to the 10th fraction. The median prescription dose was $20 \mathrm{~Gy}$ (range, 18 to $30 \mathrm{~Gy}$ ) up to a 10th fraction. The upper and lower margin of MVCT scanning was consistent during the daily treatments. The median age was 69 years (range, 33 to 86 years) and 10 patients $(24.4 \%)$ were treated postoperatively.

Results: Overall bladder volume on planning computed tomography was $139.7 \pm 92.8 \mathrm{~mL}$. Generally, post-irradiation bladder volume (POSTBV) was larger than pre-irradiation bladder volume (PREBV) $(p<0.001)$. The mean PREBV and POSTBV was reduced after 10 fraction treatments by $21.3 \%(p=0.028)$ and $25.4 \%(p=0.007)$, respectively. The MVCT-scanned body contour volumes had a tendency to decrease as the treatment sessions progressed ( $p=0.043$ at the 8th fraction and $p=0.044$ at the 10th fraction). There was a statistically significant correlation between bladder filling time and PREBV $(p=0.001)$.

Conclusion: Daily MVCT-based bladder volume assessment was feasible both intra- and inter-fractionally.
\end{abstract}

Keywords: Mega-voltage computed tomography, Radiotherapy, Pelvic neoplasms, Urinary bladder

\section{Introduction}

Urinary bladder filling is a beneficial process for sparing the small intestine during radiotherapy (RT) to the pelvic area. The achievement of consistent daily bladder expansion is one of the significant issues. Bladder volume changes can affect radiation dose distribution to the bladder itself and adjacent pelvic organs. Significant volume changes can compromise sophisticated planning data such as intensity-modulated radiotherapy (IMRT) [1-3]. Thus, the bladder volume needs to be kept consistent throughout all of the fractionated treatment sessions.

Many attempts to assess bladder volume during RT have been conducted. Ultrasonography, which is one of the useful tools for bladder volume measurement, has its restrictions in accurate set-up and consistent daily bladder volume measurements because it is a separate process during RT [4-9]. An alternative method to compensate for this problem can be a mega-voltage computed tomography (MVCT) system, which is actively used for volumetric set-up error correction during

Received 11 April 2018, Revised 22 June 2018, Accepted 31 July 2018.

Correspondence: Sei Hwan You, MD, Department of Radiation Oncology, Yonsei University Wonju College of Medicine, 20 Ilsan-ro, Wonju 26426, Korea. Tel: +82-33-741-1518, Fax: +82-33-741-1519, E-mail: ys3259@yonsei.ac.kr

(c) This is an Open Access article distributed under the terms of the Creative Commons Attribution Non-Commercial License (http://creativecommons.org/ licenses/by-nc/4.0/) which permits unrestricted non-commercial use, distribution, and reproduction in any medium, provided the original work is properly cited.

www.e-roj.org 
tomotherapy. Despite a relatively low resolution, it is effective in improving treatment accuracy by combining a scanning system with RT. Due to mega-volt energy beam characteristics, bony landmark-dependent images are the main references for error correction. However, MVCT scanning has been applied without a definite protocol. In a typical example, the scanning range is usually different for each treatment session. In addition, soft tissues such as the urinary bladder are not used so actively in set-up process. Especially, the urinary bladder needs to be utilized for set-up because the anatomic boundary is detectable on MVCT images as seen in previous pelvic tomotherapy studies [10-14].

Most assessments of bladder volume are based upon an intentional filling method, which is associated with practical limitations such as lack of objectivity and unpredictable urination timing. Thus, the validity of bladder filling requires more detailed assessment in the era of IMRT. In this study, we intended to quantify the daily bladder volume changes during the initial course of RT for pelvic malignancy using MVCT images and to assess the feasible set-up techniques with systematic MVCT application.

\section{Materials and Methods}

\section{Patient characteristics}

Between October 2014 and January 2016, 52 consecutive patients with pelvic irradiation indication were enrolled after patient approval for this prospective study. Among them, 11 patients were excluded because of treatment interruption or poor MVCT image quality and 41 patients were finally analysed. All patients received helical tomotherapy using the Hi-Art System (TomoTherapy Inc., Madison, WI, USA). None had severe urinary dysfunction initially. Their median age was 69 years (range, 33 to 86 years). Their diagnoses were prostate cancer, rectal cancer, cervix cancer, bladder cancer, and other pelvic area tumours. This study was approved by the Institutional Review Board of Yonsei University Wonju College of Medicine (No. YWMR-14-7-024).

All patients underwent simulation with supine position using computed tomography (CT) of a 3-5 mm slice thickness including the pelvis and lower abdomen. Then target delineation was drawn using Pinnacle v7.6 radiotherapy planning systems (Philips, Cleveland, OH, USA) and was optimized to reflect each disease status. RT was planned as three-dimensional RT or IMRT. Ten patients (24.4\%) were treated with postoperative RT. IMRT with a helical mode was applied to 13 patients (31.7\%) and the others were irradiated by a three-dimensional conformal method with a direct mode. MVCT analysis was performed for the initial 10 consecutive fractions of each RT course regardless of the total prescription dose focusing on the bladder volume and its dose distribution. Patient characteristics related to clinical factors are displayed in Table 1.

Table 1. Patients' characteristics $(n=41)$

\begin{tabular}{lc}
\hline \multicolumn{1}{c}{ Characteristic } & Value \\
\hline Age (yr) & $66.3 \pm 12.4$ \\
Median (range) & $69(33-86)$ \\
Sex & \\
Male & $31(75.6)$ \\
Female & $10(24.4)$ \\
Diagnosis & \\
Prostate cancer & $14(34.1)$ \\
Rectal cancer & $21(51.2)$ \\
Cervix cancer & $3(7.3)$ \\
Bladder cancer & $1(2.4)$ \\
Others & $2(4.9)$ \\
Postoperative radiotherapy & $10(24.4)$ \\
Yes & $31(75.6)$ \\
No & \\
Endorectal balloon insertion & $14(34.1)$ \\
Yes & $27(65.9)$ \\
No & \\
Radiotherapy technique & $28(68.3)$ \\
Three-dimensional conformal & $13(31.7)$ \\
Intensity-modulated &
\end{tabular}

Values are presented as mean \pm standard deviation or number (\%).

\section{Bladder dosimetry on MVCT images}

All patients were irradiated in a supine position with natural bladder filling. Patients with prostate cancer were treated with urination for 1-1.5 hours before treatment but were not strictly enforced, and no other bladder filling training was given to other patients. The MVCT scan was performed right before and after the daily irradiation. The pre-irradiation MVCT scan (PRESCAN) image was matched with the planning CT image based upon the three-dimensional bony structure followed by shifting the couch to the optimal position. After irradiation, a post-irradiation MVCT scan (POSTSCAN) was taken in the same position, in which the original reference point was maintained. Basically, the MVCT scan range was set from the sacral promontory level to the lower margin of the ischium and it was partially modified according to bladder 
volume extent. This scan range was maintained consistently from the 1st fraction to the 10th fraction for each patient's RT course for both PRESCAN and POSTSCAN. The pelvic body outline volume (POV), which includes pelvic organs, skeletal, and soft tissue, was also calculated by contouring the outermost skin area on MVCT image slices for a consistent scan range from the 1st fraction to the 10th fraction for each patient's RT course. This was also done by one radiation oncologist using the Hi-Art System for both PRESCAN and POSTSCAN and the method of volume measurement was the same as for bladder volume.

Bladder volume at the planning $\mathrm{CT}$ was measured on a kilovoltage CT image of 3-5 $\mathrm{mm}$ thickness as a routine planning process. During the RT course, it was calculated by contouring the bladder wall on every 6-mm slice of the MVCT images. This was performed by one radiation oncologist using a $\mathrm{Hi}$ Art System and the volume was measured by an operating planning process from the 1st fraction to the 10th fraction for each patient's RT course for both PRESCAN and POSTSCAN (Fig. 1). We analysed the inter-fractional variability in daily bladder volumes according to the prescription dose to the clinical target volume (CTV) and bladder dose distribution from the 1st fraction to the 10th fraction. In addition, the intra-fractional variability was assessed by comparing PRESCAN data with POSTSCAN data for each RT fraction. Planning CT-based dosimetric parameters for the initial 10 fractions are shown in Table 2.

\section{Clinical factors}

We assessed the correlations between bladder volume change and potential clinical predictors such as urinary ascorbic acid, urinary white blood cells, and urinary red blood cells along with surgical intervention, bladder filling sensation, bladder filling time, and time gap between PRESCAN and POSTSCAN. The bladder filling time was defined as the interval from the last urination to the PRESCAN time point for each fraction.

\section{Statistical analysis}

A paired t-test was used for comparison of the PRESCAN and POSTSCAN data. Surgical intervention was analyzed by independent t-test, the correlations between other clinical factors and bladder volume changes were evaluated by the Pearson correlation method. Statistical significance was defined at the $p<0.05$ level. The SPSS version 20.0 (IBM, Armonk, NY, USA) program was used for analysis.

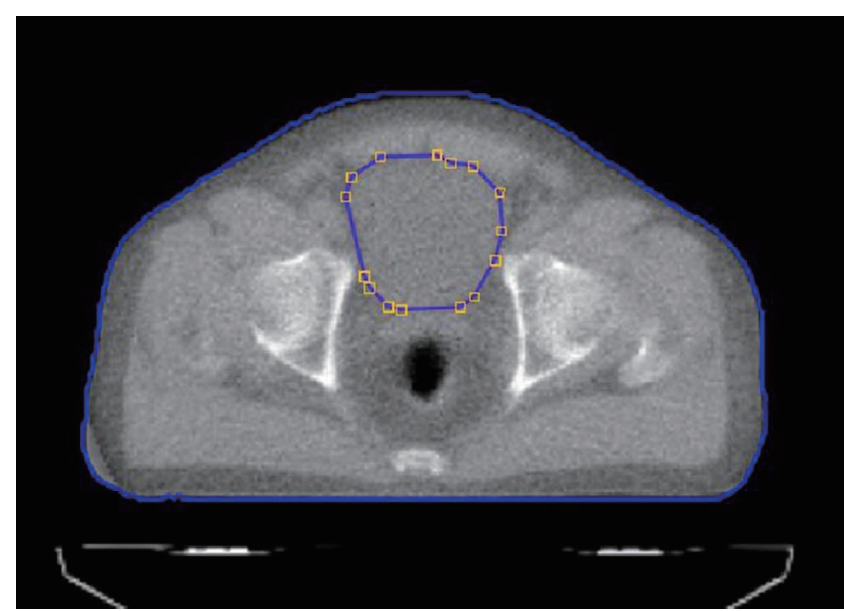

Fig. 1. An example of a mega-voltage computed tomography image and bladder contouring.

Table 2. Planning CT-based dosimetric parameters in terms of consecutive 10 fractions

\begin{tabular}{lc}
\hline \multicolumn{1}{c}{ Parameter } & Value \\
\hline Prescription dose (Gy) & $20.0(18.0-30.0)$ \\
Bladder volume (mL) & $105.9(24.9-354.5)$ \\
$\quad$ Mean & 139.7 \\
Bladder dose (Gy) & \\
Mean & $14.9(5.6-19.3)$ \\
Max & $20.3(14.4-28.4)$ \\
Min & $7.0(0.4-17.9)$ \\
\hline
\end{tabular}

Values are presented as median (range).

$\mathrm{CT}$, computed tomography.

\section{Results}

All planned bladder volume data were acquired successfully. The median prescription dose to the CTV was $20 \mathrm{~Gy}$ (range, 18 to $30 \mathrm{~Gy}$ ) for the initial 10 fractions. The mean bladder volume on planning CT was $139.7 \pm 92.8 \mathrm{~mL}$ and its mean, maximum, and minimum doses were $14.7 \pm 3.0 \mathrm{~Gy}, 20.8 \pm 2.9 \mathrm{~Gy}$, and $8.2 \pm 3.6 \mathrm{~Gy}$, respectively, up to the 10th fraction. The mean bladder filling time was $75.3 \pm 44.8$ minutes (range, 4 to 210 minutes). It took an average of $13.6 \pm 4.8$ minutes (range, 9 to 41 minutes) from PRESCAN to POSTSCAN. The bladder filling sensation was categorized into three stages of the patients' subjective estimation for the overall treatment sessions, full, moderate, and empty comprised $10.4 \%, 53.7 \%$, and $35.8 \%$, respectively. 


\section{MVCT bladder volume}

The mean PRESCAN bladder volume (PREBV) from the 1st to the 10th fraction was $140.4 \pm 90.2 \mathrm{~mL}$ (1st fraction), $111.9 \pm$ $59.2 \mathrm{~mL}$ (2nd fraction), $126.1 \pm 65.0 \mathrm{~mL}$ (3rd fraction), 124.5 $\pm 78.6 \mathrm{~mL}$ (4th fraction), $131.8 \pm 75.4 \mathrm{~mL}$ (5th fraction), 112.6 $\pm 65.6 \mathrm{~mL}$ (6th fraction), $118.1 \pm 72.7 \mathrm{~mL}$ (7th fraction), 107.6 $\pm 61.1 \mathrm{~mL}$ (8th fraction), $112.1 \pm 77.4 \mathrm{~mL}$ (9th fraction), and $110.5 \pm 63.8 \mathrm{~mL}$ (10th fraction), respectively. A mean PREBV reduction of $21.3 \%$ was observed between the 1 st and the 10th fraction $(p=0.028)$. A significant mean POSTSCAN bladder volume (POSTBV) reduction was also found between the 1 st and the 10th fraction (25.4\%, $p=0.007)$ (Fig. 2). For all treatment sessions, the POSTBV was larger than PREBV ( $p$ $<0.001$ ). Pre-irradiation surgery, irradiation technique, and bladder dosimetric parameters were not related to bladder volume changes. There was a weak relationship only between bladder filling sensation and bladder filling time (Pearson $r=$ $0.282, p=0.001$ ) (Fig. 3).

\section{Radiation influence on other clinical factors}

Mean POV values with standard deviation before and after irradiation from the 1st to the 10th fraction are displayed in Fig. 4. Unlike bladder volume (PREBV and POSTBV) change patterns, the intra-fractional POV changes were not remarkable. POSTSCAN volume showed a restrictive decrease tendency at the 8 th $(p=0.043)$ and 10th $(p=0.044)$ treatment session compared with PRESCAN volume. These volume change patterns had a strong tendency towards a correlation with pre-irradiation surgery at some treatment sessions ( $p=$ 0.057 at the $3 r d$ fraction, $p=0.069$ at the 6th fraction). For the cystitis assessment, only the planning maximum dose was moderately correlated with an increase in urine white blood cell numbers after the 10th fraction (Pearson $r=0.49, p=$ 0.011). As age increased, PREBV was found to be statistically significantly reduced, but the correlation was very weak (Pearson $r=-0.138, p=0.005)$. The other potential clinical predictors such as urinary ascorbic acid, urinary red blood cells, surgical intervention, ERB, and time gap between PRESCAN and POSTSCAN did not show a significant relationship with the change pattern of bladder volume or POV. There was no statistically significant difference in PREBV between prostate cancer $(118.23 \pm 75.22 \mathrm{~mL})$ and rectal cancer $(116.80 \pm 60.09$ $\mathrm{mL}$ ). In the case of other diseases, statistical significance could not be assessed because the number of the patients was too small.

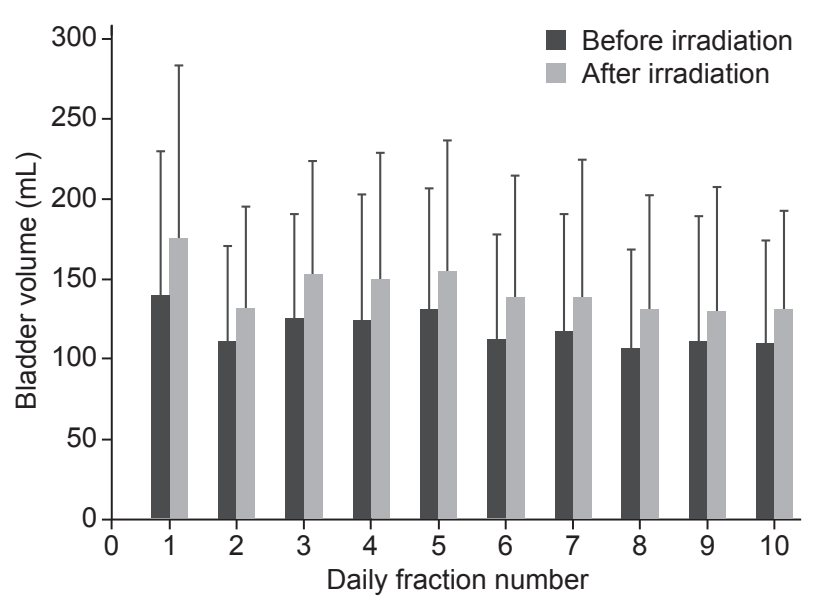

Fig. 2. Mean values of bladder volume with standard deviation before and after irradiation from the 1st to the 10th fraction.

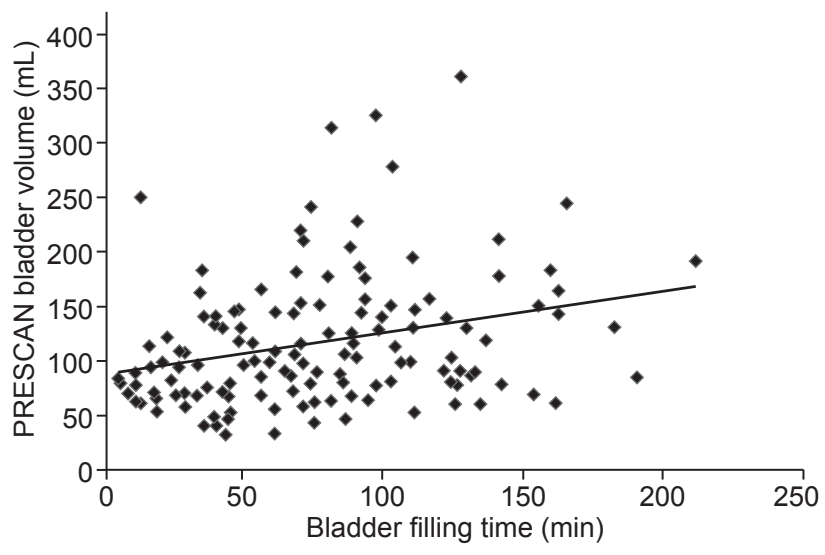

Fig. 3. Correlation of PRESCAN bladder volume and bladder filling time (Pearson $r=0.282, p=0.001$ ).

\section{Discussion and Conclusion}

The main objective of this study was to assess inter- and intra-fractional bladder volume during RT to the pelvic area. Generally, MVCT was a useful option for bladder volume assessment as well as set-up error correction. Intra-fractional bladder volume changes were found to be different for each treatment. These data suggest that the current bladder filling system should be improved and more careful approaches are needed in IMRT. For the patients in our study, the natural bladder filling method was applied without strict urination training for full bladder. Our protocol for consistent bladder 


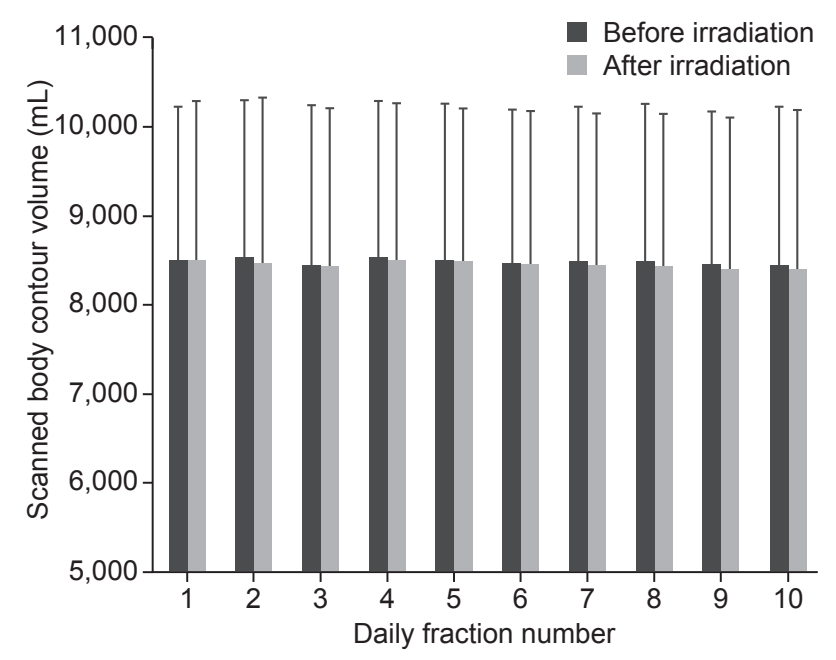

Fig. 4. Mean values of scanned pelvic body outline volume with standard deviation before and after irradiation from the 1st to the 10th fraction.

volume was based upon non-stressful urination with supine position to avoid the setup instability caused by respiratory movements. Also, elderly patients, which composed a large amount of groups in prostate cancer and rectal cancer in our study, usually had problems in urination control. Thus, despite our less strict regulation, our protocol seems to be efficient for the treatment of prostate cancer, bladder cancer, and rectal cancer, which may be supported by our MVCT results.

When using ultrasound-based measurements, patients must have their bladder volume measured separately from the RT session, which results in anatomic inconsistency. In contrast, MVCT can be a good monitoring tool because the bladder volume changes can be easily detected as an extension of the radiation treatment without a separate set-up process. Due to its relatively low resolution, MVCT application has been usually confined to bony structure comparisons in setup error corrections. However, in most cases in our study, the bladder border was easily identified on MVCT except for a few patients with unstable bowel status. Unlike ultrasonograms, MVCT could provide information on its anatomic relationship to surrounding organs as well as volumetric values of the bladder. In addition, MVCT of a consistent scan range through all treatment sessions resulted in the same volume variation pattern as that of ultrasonography in previous studies [4$7,10,15]$. Thus, the MVCT-mediated approach in our study appears to be valid.

At present, due to large inter-fractional variation, it is difficult to find a suitable protocol that ensures consistent bladder volumes $[5,7,10]$. Actually, intra-fractional volume increase is an inevitable problem. However, dosimetric and clinical effects might not be large considering the modern irradiation process in pelvic area RT except bladder cancer. Rather, other clinical factors such as urination pattern, cystitis, or adjacent tissue effect may be more influential than the bladder volume itself. On the other hand, inter-fractional variation can be an issue to pay attention to because of its clinical effects and the possibility for improvement. Despite many unknown variables, regular volume reduction is observed as the treatment session progresses $[4-7,10,15,16]$. Our study also revealed a bladder volume decline even in the early phase of the treatment course, which can be explained by a low dose effect or tissue susceptibility including cystitis or fibrosis $[17,18]$

A similar decline of inter-fractional POV might not be overlooked considering the correlation with bladder volume change. Despite an intra-fractional bladder volume increase, the corresponding intra-fractional POV had a tendency to decrease paradoxically, which was contrary to existing concepts. From these results, we can guess the possibility of a physiological response to surrounding tissue elasticity changes. This point should be discussed with along with a more systematic approach in future studies.

Subjective patient assessments or biofeedback has its limitations in controlling bladder volume consistently [6]. In our study, self-assessment for voiding and bladder filling sensation was partly reliable. However, it is not sufficient for precisely controlling bladder volume. For these reasons, a more systematic bladder filling method should be devised in the era of adaptive RT, especially for the pelvic area considering the need for whole pelvic tissue stability as well as bladder volume consistency for accurate dose delivery. Other limitations of this study might be the absence of principle identification of bladder volume decreases and the insufficient resolution of the MVCT image. Improvement of these design aspects is desirable in the future. Nevertheless, our results can be meaningful in that the method of maintaining a constant MVCT scan range was applied, demonstrating the feasibility of the standardized volumetric set-up method.

In conclusion, daily MVCT-mediated bladder and body contour volume assessment was feasible both intra- and interfractionally. We expect that MVCT can be used to evaluate the bladder volume easily and accurately, and it will be of some help for better RT. 


\section{Conflict of Interest}

No potential conflict of interest relevant to this article was reported.

\section{Acknowledgments}

This study was supported by a grant from the Korea Health Technology R\&D Project, Ministry of Health \& Welfare, Republic of Korea (No. HI10C0051 [A100054]).

\section{References}

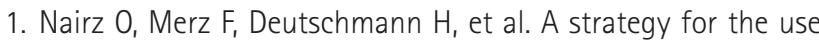
of image-guided radiotherapy (IGRT) on linear accelerators and its impact on treatment margins for prostate cancer patients. Strahlenther Onkol 2008;184:663-7.

2. Tsai CL, Wu JK, Wang CW, Hsu FM, Lai MK, Cheng JC. Using cone-beam computed tomography to evaluate the impact of bladder filling status on target position in prostate radiotherapy. Strahlenther Onkol 2009;185:588-95.

3. Moiseenko V, Liu M, Kristensen S, Gelowitz G, Berthelet E. Effect of bladder filling on doses to prostate and organs at risk: a treatment planning study. J Appl Clin Med Phys 2006;8:55-68.

4. Chang JS, Yoon $\mathrm{HI}$, Cha $\mathrm{HJ}$, et al. Bladder filling variations during concurrent chemotherapy and pelvic radiotherapy in rectal cancer patients: early experience of bladder volume assessment using ultrasound scanner. Radiat Oncol J 2013;31:41-7.

5. Ahmad R, Hoogeman MS, Quint S, Mens JW, de Pree I, Heijmen BJ. Inter-fraction bladder filling variations and time trends for cervical cancer patients assessed with a portable 3-dimensional ultrasound bladder scanner. Radiother Oncol 2008;89:172-9.

6. Stam MR, van Lin EN, van der Vight LP, Kaanders JH, Visser AG. Bladder filling variation during radiation treatment of prostate cancer: can the use of a bladder ultrasound scanner and biofeedback optimize bladder filling? Int J Radiat Oncol Biol Phys 2006:65:371-7.

7. O'Doherty UM, McNair HA, Norman AR, et al. Variability of bladder filling in patients receiving radical radiotherapy to the prostate. Radiother Oncol 2006;79:335-40.
8. Hynds S, McGarry CK, Mitchell DM, et al. Assessing the daily consistency of bladder filling using an ultrasonic Bladderscan device in men receiving radical conformal radiotherapy for prostate cancer. Br J Radiol 2011;84:813-8.

9. Orio PF 3rd, Merrick GS, Allen ZA, et al. cExternal beam radiation results in minimal changes in post void residual urine volumes during the treatment of clinically localized prostate cancer. Radiat Oncol 2009;4:26.

10. Nakamura N, Shikama N, Takahashi O, et al. Variability in bladder volumes of full bladders in definitive radiotherapy for cases of localized prostate cancer. Strahlenther Onkol 2010;186:637-42.

11. Kalz J, Sterzing F, Schubert K, Sroka-Perez G, Debus J, Herfarth K. Dosimetric comparison of image guidance by megavoltage computed tomography versus bone alignment for prostate cancer radiotherapy. Strahlenther Onkol 2009;185:241-7.

12. Fiorino C, Di Muzio N, Broggi $S$, et al. Evidence of limited motion of the prostate by carefully emptying the rectum as assessed by daily MVCT image guidance with helical tomotherapy. Int J Radiat Oncol Biol Phys 2008;71:611-7.

13. Yeung TP, Yartsev S, Rodrigues $G$, Bauman G. Evaluation of image-guidance strategies with helical tomotherapy for localised prostate cancer. J Med Imaging Radiat Oncol 2011;55:220-8.

14. Kupelian PA, Langen KM, Zeidan OA, et al. Daily variations in delivered doses in patients treated with radiotherapy for localized prostate cancer. Int J Radiat Oncol Biol Phys 2006;66:876-82.

15. Fiorino $C$, Foppiano $F$, Franzone $P$, et al. Rectal and bladder motion during conformal radiotherapy after radical prostatectomy. Radiother Oncol 2005;74:187-95.

16. Lebesque JV, Bruce AM, Kroes AP, Touw A, Shouman RT, van Herk M. Variation in volumes, dose-volume histograms, and estimated normal tissue complication probabilities of rectum and bladder during conformal radiotherapy of T3 prostate cancer. Int J Radiat Oncol Biol Phys 1995;33:1109-19.

17. Verit A, Yeni E, Unal D, Kafali H, Ozturk A, Ozardali I. Idiopathic retroperitoneal fibrosis mimicking a pelvic tumor: a case of pericystitis plastica. Yonsei Med J 2003;44:548-50.

18. Pinkawa M, Asadpour B, Gagel B, Piroth MD, Holy R, Eble MJ. Prostate position variability and dose-volume histograms in radiotherapy for prostate cancer with full and empty bladder. Int J Radiat Oncol Biol Phys 2006;64:856-61. 